\title{
Scalar kinks and fermion localisation in warped spacetimes
}

\author{
Ratna Koley * and Sayan Kar ${ }^{\dagger}$ \\ Department of Physics and Centre for Theoretical Studies \\ Indian Institute of Technology, Kharagpur 721 302, India
}

\begin{abstract}
Scalar kinks propagating along the bulk in warped spacetimes provide a thick brane realisation of the braneworld. We consider here, a class of such exact solutions of the full Einstein-scalar system with a sine-Gordon potential and a negative cosmological constant. In the background of the kink and the corresponding warped geometry, we discuss the issue of localisation of spin half fermions (with emphasis on massive ones) on the brane in the presence of different types of kink-fermion Yukawa couplings. We analyse the possibility of quasi-bound states for large values of the Yukawa coupling parameter $\gamma_{F}$ (with $\nu$, the warp factor parameter kept fixed) using appropriate, recently developed, approximation methods. In particular, the spectrum of the low-lying states and their lifetimes are obtained, with the latter being exponentially enhanced for large $\nu \gamma_{F}$. Our results indicate quantitatively, within this model, that it is possible to tune the nature of warping and the strength and form of the Yukawa interaction to obtain trapped massive fermion states on the brane, which, however, do have a finite (but very small) probability of escaping into the bulk.
\end{abstract}

\footnotetext{
* Electronic address: ratna@cts.iitkgp.ernet.in

† Electronic address : sayan@cts.iitkgp.ernet.in
} 


\section{INTRODUCTION}

Extra dimensions a la Kaluza-Klein [1, 2] have been around as an elegant theoretical construct for decades. Their inevitable presence in superstring theories [3], is by now, wellestablished. Of late, warped (nonfactorisable) spacetimes have been in vogue ever since Randall-Sundrum introduced them in the context of the now-popular braneworld models 4]. In such models, extra dimensions exist, can be compact or non-compact, but the usual schemes of 'compactification' are abandoned. The four dimensional world (the so-called 3-brane) is visualised as an embedded hypersurface in the five dimensional bulk. Effective field theories on the 3-brane are obtained without invoking 'compactification' in the usual sense of Kaluza-Klein. Further details of how this is achieved can be found in [4] or in subsequent review articles [5]. Much work has been carried out on diverse aspects of such models in the last four years. These include the attempted resolution of the heirarchy problem [4, 7], questions about the localisation of various types of fields on the brane 6], particle phenomeonology in the braneworld context [7] and cosmological consequences [8]. The actual existence of warped extra dimensions as well as a firm foundational basis for these models however, still remain open issues. Phenomenological work supported by experimental verification and simultaneous progress in formal theory (via superstrings or any other yet-tobe-discovered fundamental unified theory) will perhaps be able to provide conclusive answers about the future of the braneworld scenario.

In the early braneworld models, the bulk spacetime was assumed to have only a negative cosmological constant (anti-de Sitter space in five dimensions). Subsequently, we have had models with fields in the bulk-the simplest of them being those with a bulk scalar field dependent only on the fifth (extra) coordinate [9]. In this paper, we first consider exact solutions of the Einstein-scalar equations with a sine-Gordon potential. There exists a full solution of this system with the scalar field configuration being a kink. Such a configuration, the so-called thick brane, provides a realisation of the braneworld as a domain wall in the bulk, a fact which has been illustrated in several examples in the literature 10]. The warped background spacetime, in which the bulk sine-Gordon soliton is a self-consistent solution, turns out to have a non-constant but asymptotically negative Ricci curvature.

In the scenario mentioned above, where we have an exact solution of the full Einsteinscalar system, we address the question of localisation of fermions with emphasis on the 
massive ones. Though largely a toy model, we are able to find some interesting analytical results, which add to the only work on massive fermions in the literature [10]. To address the issue of localisation, we couple the fermion field with the scalar field through a Yukawa coupling, as has been done in many articles on fermion localisation. Subsequently, we investigate and compare the localisation effects for two different types of Yukawa couplings in the background of this scalar sine-Gordon kink in the warped line element. Known results for the massless fermions are verified. Using approximate methods, we are able to delineate which Yukawa coupling can yield better results (i.e. have more trapped states). In

addition, we make use of a new, recently developed formalism for estimating the lifetime of the states and show how the lifetime can indeed be exponentiallylarge for large values of the Yukawa coupling parameter and moderate values of the warp factor parameter. In summary, the available exact solution for the scalar field and the warp factor helps us to understand analytically, the nature of the effective potential, the trapped states, their lifetime and the comparative role of different Yukawa couplings.

\section{THE EXACT SOLUTION FOR THE WARP FACTOR AND THE SG KINK}

We begin by writing down the Lagrangian and the equations of motion for Einstein gravity with a cosmological constant, minimally coupled to a real scalar field with a potential $V(\phi)$. The action for such a system is given as :

$$
S=\int\left[\frac{1}{2 \kappa_{5}^{2}}(R-2 \Lambda)-\frac{1}{2} g^{a b} \partial_{a} \phi \partial_{b} \phi-V(\phi)\right] \sqrt{-g} d^{5} x
$$

where $g_{a b}$ is the five dimensional metric tensor with signature $(-++++)$ and $\mathrm{R}$ is the Ricci scalar. $\Lambda$ is the five dimensional cosmological constant. $\kappa_{5}^{2}=8 \pi G_{5}$ where $G_{5}$ is the five dimensional Newton constant.

We assume a line element, in the five dimensional bulk, of the form :

$$
d s^{2}=d \sigma^{2}+e^{-2 f(\sigma)} \eta_{i j} d x^{i} d x^{j}
$$

where $\sigma$ is the fifth coordinate and $f(\sigma)$ is the warp factor. The scalar field is considered to be a function of $\sigma$ only. With these ansatze the Einstein scalar system reduces to the following system of coupled, nonlinear ordinary differential equations : 


$$
\begin{aligned}
f^{\prime \prime} & =a \phi^{2} \\
f^{\prime 2} & =\frac{a}{4}\left(\phi^{\prime 2}-2 V\right)-\frac{\Lambda}{6} \\
\phi^{\prime \prime}-4 f^{\prime} \phi^{\prime} & =\frac{d V}{d \phi}
\end{aligned}
$$

where $a=\kappa_{5}^{2} / 3$. The first two of the above set are the Einstein equations and the third is the scalar field (Klein-Gordon) equation, which follows from the Einstein equations and is not independent.

The obvious question is : is there an exact analytical solution to the system of Eqns (2.3)(2.5)? For the Higgs potential, Ringeval et al [10] have numerically investigated the solutions and found kinks and the corresponding warp factors. We shall now show that the Eqns (2.3)-(2.5) can indeed be solved exactly for a sine-Gordon potential $\left(V(\phi)=B\left(1+\cos \frac{2 \phi}{\Lambda}\right)\right.$. Variants of this solution with different choices of $V(\phi)$ have appeared in the literature [12]. We comment on this at the end of this section. We now write down the solution explicitly below.

$$
\begin{gathered}
f(\sigma)=\frac{a}{\kappa_{1}} \sqrt{\frac{|\Lambda|}{6}} \ln \cosh \left(\frac{\kappa_{1}}{a} \sigma\right) \\
\phi(\sigma)=2 A \tan ^{-1}\left(\exp \frac{\kappa_{1}}{a} \sigma\right)-\frac{\pi A}{2}
\end{gathered}
$$

The constant $\mathrm{B}$ in the sine-Gordon potential is given as :

$B=\frac{|\Lambda|}{6 a^{2}}\left(a+\frac{1}{4 A^{2}}\right)$. Also $\kappa_{1}=\frac{1}{A^{2}} \sqrt{\frac{|\Lambda|}{6}}$.

It is important to note the presence of a negative cosmological constant in both the warp factor and the soliton. In addition, we emphasize that the kink/soliton is constructed in such a way that $\phi(\sigma)$ is an odd function about the origin. The second term in the expression for $\phi(\sigma)$ is required to achieve this.

This model with a bulk SG potential provides a 'thick brane' realisation of the RandallSundrum scenario where the SG field and its soliton configuration dynamically generate this domain wall configuration in the background warped geometry. In addition, as is obvious from the functional from of $f$, there is no discontinuity in the derivative of $f$ at the location of the brane. The warp factor is smooth everywhere and has all the necessary features.

The line element in the warped bulk space time is given by 


$$
d s^{2}=d \sigma^{2}+\cosh ^{-2 \nu}\left(\frac{\kappa_{1}}{a} \sigma\right) \eta_{i j} d x^{i} d x^{j}
$$

where $\nu=\frac{a}{\kappa_{1}} \sqrt{\frac{|\Lambda|}{6}}$

Notice that the metric is completely non-singular for the full domain of the fifth coordinate. It describes a space of negative Ricci curvature given by

$$
R=\frac{|\Lambda|}{6}\left[\frac{8}{a A^{2}}-\left(\frac{8}{a A^{2}}+20\right) \tanh ^{2} \frac{\kappa_{1}}{a} \sigma\right]
$$

It is straightforward to check that the above function for $\mathrm{R}$ is not always negative with a asymptotic $(\sigma \rightarrow \pm \infty)$ value of $-\frac{10}{3}|\Lambda|$. One cannot get constant negative Ricci curvature for any real value of the parameter $A$. In the above solution, the parameter $A$ is free whereas $B$ is fixed in terms of $A,|\Lambda|$ and $a$.

Furthermore, since the above $f(\sigma)$ and $\phi(\sigma)$ consistently solves the Einstein-scalar system we may want to know precisely the energy density and pressures which generates such a line element. These are given below.

$$
\begin{gathered}
\rho=-p_{x, y, z}=3 \frac{\kappa_{1}}{a} \sqrt{\frac{|\Lambda|}{6}} \operatorname{sech}^{2} \frac{\kappa_{1}}{a} \sigma-|\Lambda| \tanh ^{2} \frac{\kappa_{1}}{a} \sigma \\
p_{\sigma}=|\Lambda| \tanh ^{2} \frac{\kappa_{1}}{a} \sigma
\end{gathered}
$$

Note that, asymptotically, the stress energy is that of anti-deSitter space in five dimensions. At $\sigma=0$ (i.e. the location of the brane) we have $p_{x, y, z}=-\rho$ and $p_{\sigma}=0-$ an effective cosmological constant on the 3-brane. The energy density and pressures are plotted in Figure 2. It is worth noting that the matter stress-energy which acts as a source for the warped geometry satisfies the Null Energy Condition (NEC $\left.\rightarrow \rho+p_{i} \geq 0\right)$ though the Weak and Strong Energy Conditions (WEC and SEC) are violated (WEC $\left.\rightarrow \rho \geq 0, \rho+p_{i} \geq 0\right)$ 11].

It is worth noting that there exists related work on similar solutions for $\phi$ and $f$. In a most general case one might consider the following :

$$
f(\sigma)=A_{1} \ln (\cosh b \sigma) ; \phi(\sigma)=2 A_{2} \tan ^{-1}(\exp (b \sigma))-\frac{\pi A_{2}}{2} ; \quad V(\phi)=C+D \cos \left(2 \frac{\phi}{A_{2}}\right)
$$

Using these in the equations of motion one obtains the following constraints on $A_{1}, A_{2}$, $C, D$ and $|\Lambda|$. 


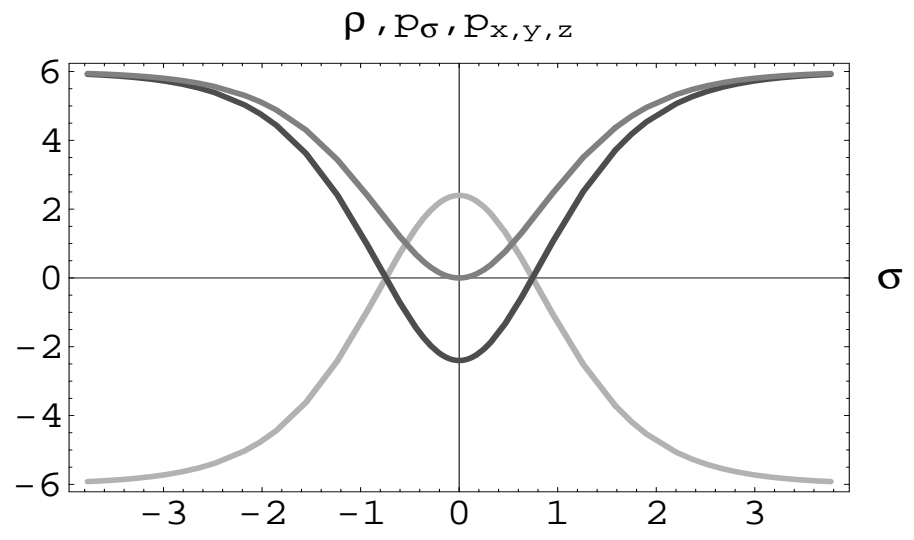

FIG. 1: Variation of the energy density $\rho$ (gray line) and pressure ( $p_{i}$ (black line) and $p_{\sigma}$ (dark gray line)) with $\sigma$ corresponding to the warped geometry given by the metric (4.3)

$$
\begin{array}{r}
A_{1}=a A_{2}^{2} ; \quad A_{2}^{2} b^{2}\left(1+4 A_{1}^{2}\right)=8 B \\
C=-\frac{A_{1}^{2} b^{2}}{a}+\frac{A_{2}^{2} b^{2}}{4}+\frac{|\Lambda|}{3} \\
D=\frac{A_{1}^{2} b^{2}}{a}+\frac{A_{2}^{2} b^{2}}{4}
\end{array}
$$

It is easy to note from the above that if $\Lambda=0$ it is not possible to have $C=D$. Various expressions for $V(\phi)$ with the corresponding solutions have been noted in the literature 12 ] though these are all for $\Lambda=0$. The solutions for $|\Lambda|=0$ and $A_{1} \neq A_{2}$ can be matched with the solutions for $|\Lambda| \neq 0$ with $A_{1}=A_{2}=2 D$, if, in the former, we assume $A_{2}-A_{1}=2 \frac{|\Lambda|}{3 a}$. This means that an 'effective' $\Lambda$ will appear within the scalar potential. However, we prefer to keep $\Lambda$ separate. As we shall see later, the mass spectrum of the localised fermions will be dependent on $\eta_{F}$ and $|\Lambda|$. It is also worth mentioning that in some of the literature on similar domain wall solutions the SG soliton is taken to be a neither-odd nor-even function. We have used a shifted version (shift along the $\phi$ axis, in order to maintain the odd character of the soliton solution and also to have $\phi=0$ at the brane location). The use of the purely odd soliton is important because, as we note in the next section, the symmetry of the effective potential for massive fermions w.r.t. the brane location is respected only if $\phi$ is purely odd. This is desirable because the physics on either side of the brane should have identical features $\left(Z_{2}\right.$ symmetry). 
In this context, one must also mention the extensive work done in the recent past on non-supersymmetric as well as supersymmetric [13], 14] domain walls in diverse models and theories.

Let us now focus on the question of localisation of massive and massless fermion fields on the 3-brane.

\section{FERMION LOCALISATION}

As with other fields such as scalars, spin one fields, gravitons, gravitinos, it is a pertinent question to ask whether spin-half fermions are/can be localised on the brane. To address this issue, one needs to assume fermions as propagating in the bulk (i.e. spinor fields functionally dependent on $\sigma$ ) in the five dimensional warped background geometry. It has been shown 6, 17] that massless fermions in a warped geometry with a RS warp factor (proportional to $\sigma)$ cannot be localised. However with additional couplings with, say, a scalar (e.g. Yukawa couplings) it is possible to obtain localised massless chiral fermions on the brane following the method developed in [18] (in 4D flat spacetime). The question of localisation of massive fermions is a problem which we shall address here for the toy model with a sine-Gordon potential and a Yukawa coupling between the fermion and the scalar fields.

The simplest set up for localisation is as follows. As mentioned earlier, one can achieve localised fermions on the brane by introducing the Yukawa coupling between fermion field and the scalar field, $\eta_{F} \bar{\Psi} \mathrm{F}(\Phi) \Psi$ where $\mathrm{F}(\Phi)$ is a function of the rescaled scalar field (i.e. $\left.\Phi=\frac{\phi}{A}\right)$. Let us first obtain the equation that governs the bulk motion of the fermions. To start with, we find that the Lagrangian for a Dirac fermion propagating in a five dimensional space with the metric (2.8), is given by

$$
\sqrt{-g} \mathcal{L}_{\text {Dirac }}=\sqrt{-g}\left(i \bar{\Psi} \Gamma^{a} \mathcal{D}_{a} \Psi-\eta_{F} \bar{\Psi} \mathrm{F}(\Phi) \Psi\right)
$$

where $g=\operatorname{det}\left(g_{a b}\right)$, is the determinant of full five dimensional metric. The matrices $\Gamma^{a}=\left(e^{f(\sigma)} \gamma^{\mu},-i \gamma^{5}\right)$ provide a four dimensional representation of the Dirac matrices in five dimensional curved space. Where $\gamma^{\mu}$ and $\gamma^{5}$ are the usual four dimensional Dirac matrices in chiral representation. The Clifford algebra in curved space, $\left\{\Gamma^{a}, \Gamma^{b}\right\}=2 g^{a b}$ is obeyed by the five dimensional gamma matrices as their counterparts follow in four dimensions. These representations have the useful property that they can induce a chiral particle theory on the 
brane [18].

The covariant derivative in 5D curved space can also be calculated for the metric given in Eqn. (2.8) [20]:

$$
\mathcal{D}_{\mu}=\left(\partial_{\mu}-\frac{1}{2} f^{\prime}(\sigma) e^{-f(\sigma)} \Gamma_{\mu} \Gamma^{4}\right) ; \quad \mathcal{D}_{4}=\partial_{\sigma}
$$

Applying the above configuration to the Eqn.(3.1) one obtains the Dirac Lagrangian in $5 \mathrm{D}$ curved spacetime in the following form

$$
\sqrt{-g} \mathcal{L}_{\text {Dirac }}=e^{-4 f(\sigma)} \bar{\Psi}\left[i e^{f(\sigma)} \gamma^{\mu} \partial_{\mu}+\gamma^{5}\left(\partial_{4}-2 f^{\prime}(\sigma)\right)-\eta_{F} \mathrm{~F}(\Phi)\right] \Psi
$$

The dimensional reduction from $5 \mathrm{D}$ to $4 \mathrm{D}$ is performed in such a way that the standard four dimensional chiral particle theory is recovered. The five dimensional spinor can be decomposed into four dimensional and fifth dimensional parts: $\Psi\left(x^{\mu}, \sigma\right)=\Psi\left(x^{\mu}\right) \xi(\sigma)$. Since the four dimensional massive fermions require both the left and right chiralities it is convenient to organise the spinors with respect to $\Psi_{L}$ and $\Psi_{R}$ which represent four component spinors living in five dimensions given by $\Psi_{L, R}=\frac{1}{2}\left(1 \mp \gamma_{5}\right) \Psi$. Hence the full 5D spinor can be split in the following way

$$
\Psi\left(x^{\mu}, \sigma\right)=\left(\Psi_{L}\left(x^{\mu}\right) \xi_{L}(\sigma)+\Psi_{R}\left(x^{\mu}\right) \xi_{R}(\sigma)\right)
$$

where we have to determine $\xi(\sigma)$ which is an eigenfunction dependent only on $\sigma$. Let us assume that the functions $\xi_{L . R}(\sigma)$ satisfy the following eigenvalue equations

$$
\begin{aligned}
& e^{-f(\sigma)}\left[\partial_{\sigma}-2 f^{\prime}(\sigma)-\eta_{F} \mathrm{~F}(\Phi)\right] \xi_{R}(\sigma)=-m \xi_{L}(\sigma) \\
& e^{-f(\sigma)}\left[\partial_{\sigma}-2 f^{\prime}(\sigma)+\eta_{F} \mathrm{~F}(\Phi)\right] \xi_{L}(\sigma)=m \xi_{R}(\sigma)
\end{aligned}
$$

The full 5D action then reduces to the standard four dimensional action for the massive chiral fermions (with mass $m$ ), when integrated over the extra dimension [10], provided (a) the above equations are satisfied by the bulk fermions and (b) the following orthonormality conditions are obeyed.

$$
\begin{array}{r}
\int_{-\infty}^{\infty} e^{-3 f(\sigma)} \xi_{L_{m}} \xi_{L_{n}} d \sigma=\int_{-\infty}^{\infty} e^{-3 f(\sigma)} \xi_{R_{m}} \xi_{R_{n}} d \sigma=\delta_{m n} \\
\int_{-\infty}^{\infty} e^{-3 f(\sigma)} \xi_{L_{m}} \xi_{R_{n}} d \sigma=0
\end{array}
$$


The Yukawa coupling between the scalar and the fermion, with the kink solution for the scalar, is necessarily like an effective, variable, 5D mass for the fermions [6]. This is largely responsible for generating the massive fermion modes in four dimensions. The dynamical features of the model can thus be obtained from the solutions of the eigenvalue equations (3.5) and (3.6).

Let us first focus on massless (i.e. $m=0$ ) fermions for a Yukawa coupling of the form $\eta_{F} \bar{\Psi} \Phi \Psi$ (i.e. $\left.\mathrm{F}(\Phi)=\Phi\right)$. In this case Eqn. (3.5) and (3.6) reduce into two decoupled equations. The solutions of which are asymptotically in the following form

$$
\begin{aligned}
& \xi_{L}(\sigma)=e^{-\left(\frac{\eta_{F} \pi}{2}-2 \sqrt{\frac{|\Lambda|}{6}}\right)|\sigma|} \\
& \xi_{R}(\sigma)=e^{\left(\frac{\eta_{F} \pi}{2}+2 \sqrt{\frac{|\Lambda|}{6}}\right)|\sigma|}
\end{aligned}
$$

Eqn. (3.9) yields the localisation of left chiral fermions on the brane so long as, $\eta_{F}>$ $\frac{4}{\pi} \sqrt{\frac{\mid \Lambda \Lambda}{6}}$. We can as well achieve the bounded right chiral states by considering the interaction with an anti-kink sine-Gordon profile.

We now turn our attention towards the massive fermions i.e. $m \neq 0$. Defining $\tilde{\xi}_{L . R}(\sigma)=$ $e^{-\frac{3}{2} f(\sigma)} \xi_{L . R}(\sigma)$ and making use of $\partial_{\sigma} \tilde{\xi}_{R}(\sigma), \tilde{\xi}_{R}(\sigma)$ from Eqn. (3.5) and (3.6) we obtian the following second order, decoupled equation for the left chiral fermions whereas the right chiral states can be completely defined from the later equation with a prior knowledge to $\tilde{\xi}_{L}(\sigma)$ :

$$
\begin{gathered}
{\left[\partial_{\sigma}^{2}-2 f^{\prime}(\sigma) \partial_{\sigma}+m^{2} e^{2 f(\sigma)}+\frac{3}{4} f^{\prime 2}(\sigma)-\eta_{F}^{2} \mathrm{~F}(\Phi)^{2}\right.} \\
\left.-\eta_{F} f^{\prime}(\sigma) \mathrm{F}(\Phi)+\eta_{F} \frac{\mathrm{dF}}{\mathrm{d} \Phi} \Phi^{\prime}(\sigma)-\frac{f^{\prime \prime}(\sigma)}{2}\right] \tilde{\xi}_{L}(\sigma)=0 \\
\tilde{\xi}_{R}(\sigma)=\frac{e^{-f(\sigma)}}{m}\left[\partial_{\sigma}+\eta_{F} \mathrm{~F}(\Phi)-\frac{f^{\prime}(\sigma)}{2}\right] \tilde{\xi}_{L}(\sigma)
\end{gathered}
$$

We note that the equation satisfied by $\tilde{\xi}_{L}(\sigma)$ (Eqn. 3.11) is a second order differential equation that looks like a time-independent Schrödinger equation (more precisely like a 'zero energy' Schrödinger equation), when recast in the following form

$$
\partial_{\sigma}^{2} \hat{\xi}_{L}(\sigma)+\left[m^{2} e^{2 f(\sigma)}+\frac{f^{\prime \prime}(\sigma)}{2}+\eta_{F} \frac{\mathrm{dF}}{\mathrm{d} \Phi} \Phi^{\prime}(\sigma)-\left(\eta_{F} \mathrm{~F}(\Phi)+\frac{f^{\prime}(\sigma)}{2}\right)^{2}\right] \hat{\xi}_{L}(\sigma)=0
$$


where, $\hat{\xi}_{L}(\sigma)=e^{-f(\sigma)} \tilde{\xi}_{L}(\sigma)$.

We are interested in localisation of massive fermions around the brane. The effective potential for the left chiral fermions obtained from the Eqn. (3.13) should have a minimum at the brane in order to ensure the confinement of fermions. The $Z_{2}$ symmetry of the bulk spacetime with respect to the position of the brane (at $\sigma=0$ ) puts a restriction on the choice of the form of the Yukawa coupling. If we demand such a symmetry for the effective potential $F(\Phi)$ should necessarily be an odd function of $\Phi$. For example, if we consider the coupling as $\eta_{F} \bar{\Psi} \Phi \Psi$ (i.e. $\mathrm{F}(\Phi)=\Phi$ ) one obtains a symmetric effective potential given by

$$
\begin{aligned}
V_{e f f}(y) & =\left(2 \gamma_{F} \tan ^{-1}\left(e^{\hat{y}}\right)-\frac{\pi \gamma_{F}}{2}+\frac{1}{2} \sqrt{\frac{1}{6}} \tanh (\hat{y})\right)^{2}-\frac{\kappa_{1}}{2 a} \sqrt{\frac{1}{6|\Lambda|}} \operatorname{sech}^{2}(\hat{y}) \\
& -\frac{\gamma_{F}}{\sqrt{|\Lambda|}} \frac{\kappa_{1}}{a} \operatorname{sech}(\hat{y})-\mu^{2} \cosh ^{2 \nu}(\hat{y})
\end{aligned}
$$

The above relation is written using a dimensionless variable and dimensionless parameters :

$$
y=\sigma \sqrt{|\Lambda|} \quad ; \quad \mu=\frac{m}{\sqrt{|\Lambda|}} \quad ; \quad \gamma_{F}=\frac{\eta_{F}}{\sqrt{|\Lambda|}}
$$

and for simplicity $\frac{\kappa_{1}}{a \sqrt{|\Lambda|}} y$ has been renamed as $\hat{y}$.

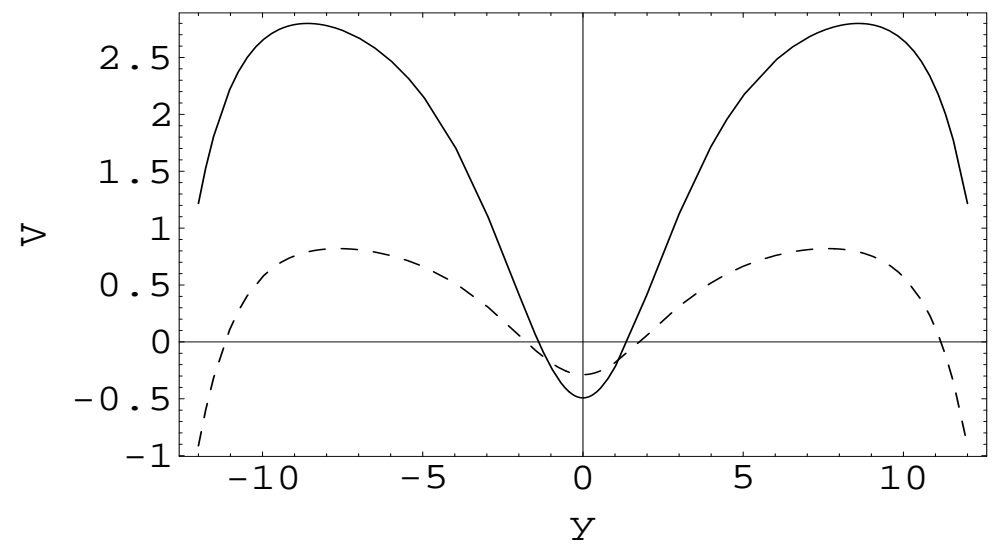

FIG. 2: For $\mathrm{F}(\Phi)=\Phi$, the effective potential $\mathrm{V}$ is plotted as a function of the rescaled extra dimension, y for different values of $\gamma_{F}$. The black curve corresponds to $\gamma_{F}=1$, whereas the dashed curve represents $\mathrm{V}$ for $\gamma_{F}=\frac{1}{2}$. The parameters are chosen as $\nu=1$ and $\Lambda=-6$.

Similarly, for $\mathrm{F}(\Phi)=\sin \Phi$ the $Z_{2}$ symmetric effective potential turns out to be : 


$$
V_{e f f}(y)=\left(\gamma_{F}+\frac{1}{2} \sqrt{\frac{1}{6}}\right)^{2} \tanh ^{2}(\hat{y})-\frac{\kappa_{1}}{a \sqrt{|\Lambda|}}\left(\gamma_{F}+\frac{1}{2} \sqrt{\frac{1}{6}}\right) \operatorname{sech}^{2}(\hat{y})-\mu^{2} \cosh ^{2 \nu}(\hat{y} \mid 3
$$

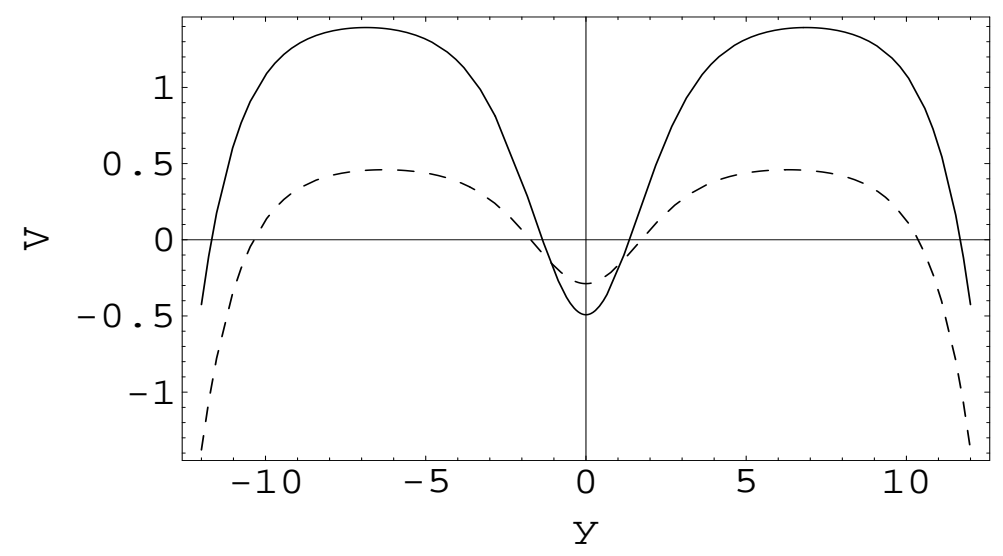

FIG. 3: The effective potential $\mathrm{V}$ is plotted as a function of the rescaled extra dimension, y with different values of $\gamma_{F}$ for $\mathrm{F}(\Phi)=\sin \Phi$. The black curve corresponds to $\gamma_{F}=1$, whereas the dashed curve represents $\mathrm{V}$ for $\gamma_{F}=1 / 2$. The parameters are chosen as $\nu=1$ and $\Lambda=-6$.

As mentioned before, the crucial point here is the antisymmetric nature of the function $\mathrm{F}(\Phi)$ under the transformation $\Phi \rightarrow-\Phi$. It can be be easily shown that a symmetric $\mathrm{F}(\Phi)$, such as $\cos \Phi, \Phi^{2}$ etc. yields an asymmetric effective potential. For the two different types of Yukawa interactions the effective potential acting on the left chiral fermions is shown in Fig.(3) and (4) for varying values of $\gamma_{F}$. We have chosen a value of $\mu$ which may not correspond to the bound state. All the plots show a minimum at $y=0$, which indicates that the massive fermions can be trapped on the brane. We notice, in addition, that the potential is, in general symmetric about the point $y=0$. Furthermore, it is worth noting that the value of the potential at $y=0$ in both the cases is given by :

$$
V_{e f f}(y=0)=-\frac{\kappa_{1}}{a} \frac{1}{\sqrt{|\Lambda|}}\left(\gamma_{F}+\frac{1}{2 \sqrt{6}}\right)-\mu^{2}
$$

However, in either case, with increasing $\gamma_{F}$, the depth of the well at the location of the brane becomes larger, demonstrating that more trapped states are possible for stronger Yukawa coupling. Additionally, the depth also increases for small $\nu$ (or large $p$ ). On the other hand, for small $\gamma_{F}$ the depth is less. and we expect very few trapped states (maybe none). The nature of the potential has prompted researchers to name it as a 'volcano' 
potential. A fair amount of work related to bound states and resonances in such volcano potentials has appeared in the literature in the recent past [15].

We can obtain an exact solution of the equation (3.13) for $\gamma_{F}=0\left(\hat{\xi}_{L} \sim \frac{\exp \left(i \sqrt{A_{2}} r\right)}{\left(1+r^{2}\right)^{\frac{1}{4}}}\right.$ where $r$ and $A_{2}$ are defined later). However, this turns out to be an asymptotically growing function (recall that $\tilde{\xi}=e^{f(\sigma)} \hat{\xi}$ and we need to consider $\tilde{\xi}$ or $\xi$ and not $\hat{\xi}$ ). The function $\tilde{\xi}_{L}$ is also not normalisable according to the orthonormality conditions given in Eqn. (3.7). Based on all the above arguments we may therefore conclude that the confinement of massive fermions on the brane cannot be achieved without a strong Yukawa interaction. Moreover the bound on

the coupling constant, $\eta_{F}>\frac{4}{\pi} \sqrt{|\Lambda| / 6}$, required for the confinement of massless fermions also suggests that a large $\gamma_{F}$ is crucial for the existence of massive bound states on the brane.

We may also view the appearance and total number of bound states as a result of a competition between gravity and the Yukawa interaction. Without gravity, in a flat five dimensional spacetime the effective potential reduces to the Pösch-Teller potential, for which exact bound state solutions [19] exist. But without the Yukawa interaction, we tend to get very few bound states and mostly quasilocalised ones which may not be normalisable. Thus, the presence of gravity, in some sense, destroys the bound states which were there and therefore, a strong Yukawa interaction is necessary in order to get them back. We shall now discuss these issues in some more detail.

\section{A. The mass spectrum of the low-lying states for $\mathbf{F}(\Phi)=\Phi$ and $\sin \Phi$}

We expand the effective potential about $y=0$ and retain terms upto order $y^{2}$. This is the harmonic oscillator approximation in the neighborhood of the 3-brane. The second order differential equation for left chiral fermions in both the cases turns out to be :

$$
\partial_{y}^{2} \hat{\xi}_{L}(y)+\left[\mu^{2}-C_{1}-\frac{1}{2} k y^{2}\right] \hat{\xi}_{L}(y)=0
$$

where we have for $\mathrm{F}(\Phi)=\Phi$ :

$$
\begin{array}{r}
C_{1}=-\left(\gamma_{F}+\frac{1}{2 \sqrt{6}}\right) \frac{\kappa_{1}}{a \sqrt{|\Lambda|}} \\
C_{2}=\left(\gamma_{F}+\frac{1}{2 \sqrt{6}}\right)^{2}\left(\frac{\kappa_{1}}{a \sqrt{|\Lambda|}}\right)^{2}+\left(\frac{\gamma_{F}}{2}+\frac{1}{2 \sqrt{6}}\right)\left(\frac{\kappa_{1}}{a \sqrt{|\Lambda|}}\right)^{3}
\end{array}
$$




$$
k=2\left(C_{2}-\mu^{2} \frac{\kappa_{1}}{a} \frac{1}{\sqrt{6|\Lambda|}}\right)
$$

and for $\mathrm{F}(\Phi)=\sin \Phi, C_{1}$ is the same as before and $C_{2}$ is given by :

$$
C_{2}=\left(\gamma_{F}+\frac{1}{2 \sqrt{6}}\right)^{2}\left(\frac{\kappa_{1}}{a \sqrt{|\Lambda|}}\right)^{2}+\left(\gamma_{F}+\frac{1}{2 \sqrt{6}}\right)\left(\frac{\kappa_{1}}{a \sqrt{|\Lambda|}}\right)^{3}
$$

From the harmonic oscillator approximation we can estimate the allowed energy levels and thereby find out the allowed fermion mass spectrum. The full five dimensional function $\Psi\left(x^{\mu}, \sigma\right)$ will have a definite parity because of the overall symmetry of the problem under $\sigma \rightarrow-\sigma$. Thus the right and left chiral wavefunctions will be either odd or even. We note here that only the even states will survive in the spectrum because of the required matching of the wave-function and its derivatives at the brane location (see a discussion on this in 10]). The possible values of $\mu^{2}$ are hidden in the expression :

$$
\mu_{n}^{2}=C_{1}+(2 n+1) \sqrt{C_{2}-\mu_{n}^{2} \frac{\kappa_{1}}{a} \frac{1}{\sqrt{6|\Lambda|}}}
$$

where $n=0,2,4 \ldots$.

The discrete mass spectrum for the trapped fermions can be obtained clearly from the following relations

For, $F(\Phi)=\Phi$ :

$$
\mu_{n}^{2}=-\bar{\gamma}_{F} \frac{1}{\nu \sqrt{6}}-\frac{(2 n+1)^{2}}{12 \nu}+\left(n+\frac{1}{2}\right) \sqrt{\left(\frac{2 n+1}{6 \nu}\right)^{2}+\frac{2 \bar{\gamma}_{F}}{3 \sqrt{6} \nu^{2}}+\frac{2 \bar{\gamma}_{F}^{2}}{3 \nu^{2}}+\frac{\bar{\gamma}_{F}}{3 \sqrt{6} \nu^{3}}+\frac{1}{36 \nu^{3}}}
$$

For, $F(\Phi)=\sin \Phi$ :

$$
\mu_{n}^{2}=-\bar{\gamma}_{F} \frac{1}{\nu \sqrt{6}}-\frac{(2 n+1)^{2}}{12 \nu}+\left(n+\frac{1}{2}\right) \sqrt{\left(\frac{2 n+1}{6 \nu}\right)^{2}+\frac{2 \bar{\gamma}_{F}}{3 \sqrt{6} \nu^{2}}+\frac{2 \bar{\gamma}_{F}^{2}}{3 \nu^{2}}+\frac{2 \bar{\gamma}_{F}}{3 \sqrt{6} \nu^{3}}}
$$

where $\bar{\gamma}_{F}=\gamma_{F}+\frac{1}{2 \sqrt{6}}$.

Notice the presence of the two parameters $\gamma_{F}$ and $\nu$. For $\gamma_{F}=0$ we find that the higher states quickly approach the continuum. This is evident from the plot of $\mu_{n}^{2}$ versus $n$ in Fig. (5) which shows that the difference between $\mu_{n}^{2}$ and $\mu_{n+1}^{2}$ tends to saturate after the first 


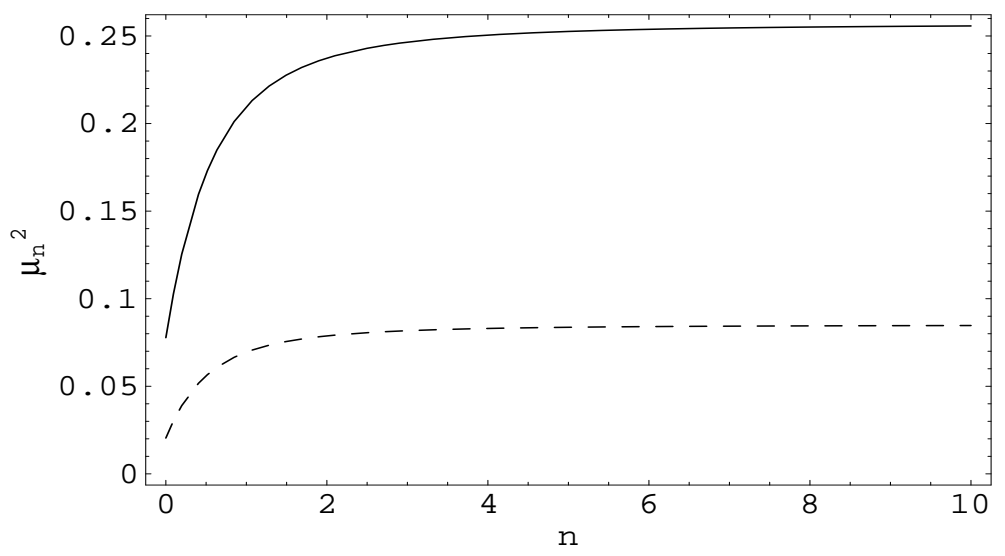

FIG. 4: The plot shows the variation of $\mu_{n}^{2}$ vs. $\mathrm{n}$ as given in Eqn. (5.34). Mass spectrum saturates very quickly for $\gamma_{F}=0$, which has been shown for $\nu=1 / 2$ (dashed curve) and 1 (continuous curve). The discrete levels reach to the continuum after a very few modes.

few states. This behaviour persists for nonzero but small $\gamma_{F}$ for which $\mu_{n}^{2}$ goes as $\sqrt{\gamma_{F}}$. However, for large $\gamma_{F}$ the $\mu_{n}^{2}$ goes as $\gamma_{F}$ and more bound states become available within the well.

1. Analysis for $\gamma_{F}>>1$ and $F(\Phi)=\Phi$

We now look for bounds on the trapped masses and the number of localised states in the limit $\gamma_{F}>>1$. The mass spectrum for this case takes the form :

$$
\mu_{n}^{2}=\frac{2 n \gamma_{F}}{\nu \sqrt{6}}
$$

There is an upper limit on the mass of the fermions which are trapped by the effective potential (3.14) because it is necessary to have a potential barrier in order to have bound states. Let us check the asymptotic behaviour of $V_{\text {eff }}$. For large values of the extra dimension the effective potential reduces to :

$$
V_{e f f} \sim-\left(\mu^{2} e^{\frac{2 y}{\sqrt{6}}}-\left(\frac{\gamma_{F} \pi}{2}+\frac{1}{2 \sqrt{6}}\right)^{2}\right)
$$

To have a potential which is a continuous function of $y$ one can match the approximate effective potentials given in eqn. (3.28) and (3.37) and obtain the point of continuity to be at $y=y_{m} \sim \frac{\pi}{2} \frac{a \sqrt{|\Lambda|}}{\kappa_{1}}$. One finds the maximum value of trapped mass from the expression 
(3.37) using the following criterion for the vanishing of the barrier :

$$
V_{e f f}\left(\mu_{\max }, y_{m}\right) \sim 0
$$

Thus for $\gamma_{F}>>1$, the maximum possible mass of the bound state is :

$$
\mu_{\max }=\frac{\gamma_{F} \pi}{2} \operatorname{Exp}\left[-\frac{\pi a}{2 \kappa_{1}} \sqrt{\frac{|\Lambda|}{6}}\right]=\frac{\gamma_{F} \pi}{2} \exp -\left(\frac{\pi}{2} \nu\right)
$$

For example, choosing the parameters as given in Fig. (3) the value of $\mu_{\max } \sim 0.33 \gamma_{F}$. The maximum number of discrete massive states localised around the brane is obtained easily from eqn. (3.36) by introducing an extra $\frac{1}{2}$ factor to account for the even states only. For a particular choice of the coupling constant and suitable range of parameters the value of $\mathrm{n}_{\max }$ can be calculated from the integer obtained by the following relation :

$$
\mathrm{n}_{\max }=\operatorname{Int}\left[\frac{1}{8}\left(\frac{\gamma_{F} \pi^{2}}{2} \frac{a \sqrt{|\Lambda|}}{\kappa_{1}} \operatorname{Exp}\left[-\frac{\pi a}{\kappa_{1}} \sqrt{\frac{|\Lambda|}{6}}\right]\right)\right]=\operatorname{Int}\left[\frac{\sqrt{6} \gamma_{F} \pi^{2}}{16} \nu \exp -(\pi \nu)\right]
$$

2. Analysis for $\gamma_{F}>>1$ and $F(\Phi)=\sin \Phi$

Let us now look at the case with $F(\Phi)=\sin \Phi$. The mass spectrum for very strong Yukawa coupling reduces to the form given in Eqn. (3.36). Far away from the brane the Effective potential turns out to be

$$
V_{\text {eff }} \sim-\left(\mu^{2} e^{\frac{2 y}{\sqrt{6}}}-\left(\gamma_{F}+\frac{1}{2 \sqrt{6}}\right)^{2}\right)
$$

Following similar steps, one can easily obtain the potential barrier just disappears at the location, $y=\frac{a \sqrt{|\Lambda|}}{\kappa_{1}}$ along the extra dimension. Applying the matching condition given in Eqn. (3.38) one can readily obtain $\mu_{\max }$ and $\mathrm{n}_{\max }$, which turn out to be :

$$
\begin{aligned}
& \mu_{\text {max }}=\gamma_{F} \operatorname{Exp}\left[-\frac{a}{\kappa_{1}} \sqrt{\frac{|\Lambda|}{6}}\right]=\gamma_{F} \exp (-\nu) \\
& \mathrm{n}_{\max }=\operatorname{Int}\left[\left(\gamma_{F} \frac{a \sqrt{|\Lambda|}}{4 \kappa_{1}} \operatorname{Exp}\left[-\frac{2 a}{\kappa_{1}} \sqrt{\frac{|\Lambda|}{6}}\right]\right)\right]=\operatorname{Int}\left[\frac{\sqrt{6} \gamma_{F}}{4} \nu \exp (-2 \nu)\right]
\end{aligned}
$$

In Table (II) we show some values of $\mu_{\max }$ and $n_{\max }$ with increasing strength of Yukawa interaction $\left(\gamma_{F}\right)$ and varying warp-factor parameter $\nu$. 


\begin{tabular}{|c|c|c|c|c|c|c|c|}
\hline \multirow{2}{*}{$\begin{array}{c}\text { Yukawa } \\
\text { Coupling }\end{array}$} & \multirow{2}{*}{$\begin{array}{c}\text { Warping } \\
\text { Parameter, } \nu\end{array}$} & \multicolumn{2}{|c|}{$\gamma_{F}=50$} & \multicolumn{2}{c|}{$\gamma_{F}=100$} & \multicolumn{3}{c|}{$\gamma_{F}=150$} \\
\cline { 2 - 9 } & $\mu_{\max }$ & $n_{\max }$ & $\mu_{\max }$ & $n_{\max }$ & $\mu_{\max }$ & $n_{\max }$ \\
\hline \multirow{3}{*}{$F(\Phi)=\Phi$} & 0.5 & 35.81 & 8 & 71.62 & 16 & 107.43 & 24 \\
\cline { 2 - 9 } & 1 & 16.33 & 3 & 32.65 & 7 & 48.98 & 10 \\
\cline { 2 - 9 } & 1.25 & 11.03 & 2 & 22.06 & 4 & 33.09 & 6 \\
\hline \multirow{3}{*}{$F(\Phi)=\sin \Phi$} & 2 & 3.39 & 0 & 6.78 & 0 & 10.18 & 1 \\
\cline { 2 - 9 } & 1 & 30.32 & 6 & 60.65 & 11 & 90.98 & 17 \\
\cline { 2 - 9 } & 1.5 & 18.39 & 4 & 36.79 & 8 & 55.18 & 12 \\
\hline
\end{tabular}

TABLE I: The maximum bound state mass, $\mu_{\max }$ and number of states, $n_{\max }$ are tabulated using $\nu=0.5,1$ and 1.25 for increasing values of $\gamma_{F}$ for the two different types of Yukawa coupling. As the coupling becomes stronger the value of $\mu_{\max }$ and $n_{\max }$ increases, which imply that the possibility of localisation is more pronounced for larger $\gamma_{F}$.

From the above numerical values for $\mu_{\max }$ and $n_{\max }$ we can make some comparative statements on the role of the two different Yukawa couplings.

(i) Equating the $\mu_{\max }$ or $n_{\max }$ for the two Yukawa couplings we obtain $\nu=.8$. Thus for $\nu<.8$ the $\Phi$ coupling has a chance of having more bound states compared to the sin $\Phi$ one. The converse is true for $\nu>$.8. Note that for $\nu=1$ (which is close to 0.8) the difference between the values of $\mu_{\max }$ and $n_{\max }$ for the $\Phi$ and $\sin \Phi$ couplings is small.

(ii) In the $F[\Phi]=\Phi$ case, the factor of $\frac{\pi}{2}$ in the expressions for $\mu_{\max }$ and $n_{\max }$ and a corresponding factor of one for the $F[\Phi]=\sin \Phi$ case seems to suggest the following generalisation for arbitrary $F[\Phi]$ :

$$
\begin{array}{r}
\mu_{\text {max }}=\gamma_{F}\{F[\Phi(\infty)]\} \exp (-\{F[\Phi(\infty)]\} \nu) \\
\mathrm{n}_{\text {max }}==\operatorname{Int}\left[\frac{\sqrt{6} \gamma_{F}}{4}(\{F[\Phi(\infty)]\} \exp (-\{F[\Phi(\infty)]\} \nu))^{2}\right]
\end{array}
$$

where $\Phi(\infty)$ is the asymptotic value of the kink profile, which, in our case here is $\pi / 2$. Thus, in addition to the parameters $\gamma_{F}$ and $\nu$, one can tune the number of bound states 
using different choices for $F[\Phi]$ too.

(iii) Also note that for $\nu=2$ or larger the $n_{\max }$ and $\mu_{\max }$ both become relatively smaller. However, as we shall see later, the lifetime of these states (with large $\gamma_{F}$ and $n u \geq 2$ ) is exponentially large. Therefore, they seem to be better candidates for describing localised massive fermions.

\section{BEYOND THE H.O. APPROXIMATION, THE LIFETIME OF QUASI- BOUND STATES}

In the above section we dealt with the harmonic oscillator approximation to get the spectrum of the lowest states. The spectrum however will be corrected by the presence of higher order terms. In particular, as we shall see below, the energy eigenvalues are in general complex with the imaginary part being related to the lifetime of the quasibound state.

To analyse this in some generality let us go back to the original Schrodinger like equation written using the variable $\sigma$. One may easily recast things using $y$ or $\hat{y}$. Expanding all terms in Taylor series upto order $\sigma^{4}$ we obtain

$$
\partial_{\sigma}^{2} \hat{\xi}_{L}(\sigma)+\left[m^{2}+\left.g^{\prime}\right|_{\sigma=0}-\left\{a_{1} \sigma^{2}-a_{2} \sigma^{4}\right\}\right] \hat{\xi}_{L}(\sigma)=0
$$

where,

$$
\begin{aligned}
& a_{1}=\left[m^{2} f^{\prime \prime}-g^{\prime 2}+\frac{g^{\prime \prime \prime}}{2}\right]_{\sigma=0} \\
& a_{2}=\left[m^{2}\left(\frac{f^{\prime \prime \prime \prime}}{12}+\frac{f^{\prime \prime 2}}{2}\right)+\frac{g^{\prime \prime \prime \prime \prime}}{4 !}-\frac{g^{\prime} g^{\prime \prime \prime}}{3}\right]_{\sigma=0}
\end{aligned}
$$

In the above expressions the prime denotes derivative with respect to $\sigma$ and $g(\sigma)=$ $\eta_{F} F(\Phi)+f^{\prime}(\sigma) / 2$. For the constant $a_{1}$ and $a_{2}$ positive we can treat the potential as harmonic oscillator potential plus a perturbation term proportional to $\sigma^{4}$ if the parameter $a_{2}$ can be assumed as very small compared to $a_{1}$. Given the symmetry of the system under the transformation $\sigma \rightarrow-\sigma$ we put a restriction on the Yukawa coupling term $F(\Phi)$ such that

the even order derivatives of $g(\sigma)$ vanish at the location of the brane. In addition, keeping in mind the problem in hand, the odd order derivatives of $\mathrm{f}(\sigma)$ is also assumed to be zero at the brane location. It is worth noting that under these assumptions and within the 
approximation the potential has a volcano profile. Keeping further terms will not alter the nature of the potential to a very great extent. Let us now find out the lifetime of the quasibound state for the particular choice of coupling $F(\Phi)=\sin \Phi$. All the above mentioned properties are satisfied by the specific $\mathrm{f}(\sigma)$ and $\mathrm{g}(\sigma)$ discussed earlier in this article. We assume $|\Lambda|=6$ for convenience. In particular, we have,

$$
g(\sigma)=\left(\eta_{F}+\frac{1}{2}\right) \tanh \left(\frac{\sigma}{\nu}\right)
$$

Using the general formulae for $a_{1}$ and $a_{2}$ we obtain:

$$
\begin{array}{r}
a_{1}=\left[-\frac{m^{2}}{\nu}+\frac{\bar{\eta}_{F}^{2}}{\nu^{2}}+\frac{\bar{\eta}_{F}}{\nu^{3}}\right] \\
a_{2}=\left[\frac{m^{2}}{2 \nu^{2}}\left(1+\frac{1}{3 \nu}\right)+\frac{2}{3} \frac{\bar{\eta}_{F}}{\nu^{4}}\left(\frac{1}{\nu}+\bar{\eta}_{F}\right)\right]
\end{array}
$$

where $\left(\eta_{F}+\frac{1}{2}\right)$ is replaced by $\bar{\eta}_{F}$. It is useful to mention here that factors of $s q r t|\Lambda| / 6$ which have been taken to be one with the choice $|\Lambda|=6$ provide the correct dimensions for each term in the above and in the subsequent discussions. We note that in the range of the warping parameter $\nu \geq 2$ the perturbation constant $a_{2}$ is reasonably small compared to a. One can see this by further approximating the potential for moderately large $\nu$ and large $\bar{\eta}_{F}$. Ignoring terms proportional to $m^{2}$ and $\bar{\eta}_{F}$ we find that the effective potential can be written as :

$$
V(\sigma)=\frac{\bar{\eta}_{F}^{2}}{\nu^{2}}\left(\sigma^{2}-\frac{2}{3 \nu^{2}} \sigma^{4}\right)
$$

Using the above approximate potential we can use the method developed in [16] for the calculation of the lifetime, $\tau=-\frac{1}{2 \operatorname{ImE}}$ (where ImE is a small negative quantity). We briefly summarize their method below.

The imaginary part $\operatorname{ImE}$ is given by the formula :

$$
\operatorname{ImE}=\frac{1}{\mathrm{i}} \frac{\int_{-\infty}^{\infty}\left(\xi \frac{\mathrm{d}^{2} \xi^{*}}{\mathrm{~d} \sigma^{2}}-\xi^{*} \frac{\mathrm{d}^{2} \xi}{\mathrm{d} \sigma^{2}}\right) \mathrm{d} \sigma}{\int_{-\infty}^{\infty}|\xi(\sigma)|^{2} \mathrm{~d} \sigma}
$$

The denominator is calculated using the zeroth order wavefunction (harmonic oscillator) in Rayleigh Schrödinger perturbation theory. In such an approximation we assume that the 
dominant contribution to the norm comes from the small values of $\sigma$. For the numerator, we use the WKB method with the assumption that the probability current is dominated by the contribution of the wave function in the classically forbidden region. Same normalisation for the small and large $\sigma$ solution has been guaranteed by the asymptotic matching of the respective solutions in these regions.

It is easy to show (following [16] ) that if $\bar{\eta}_{F}$ is large and $\nu \geq 2$ the lifetime of the metastable lowest state of the harmonic oscillator goes as

$$
c \tau \approx \frac{\nu^{\frac{1}{2}}}{\bar{\eta}_{F}^{\frac{3}{2}}} \exp \left(\bar{\eta}_{F} \nu\right)
$$

From the above formula it is clear that the lifetime is exponentially enhanced for large $\bar{\eta}_{F} \nu$. Note that $\nu \geq 2$ is required in order to treat the $\sigma^{4}$ term as a perturbation. If $\bar{\eta}_{F} \nu \tilde{>} 50$ then the lifetime will be close to the age of the universe $\left(10^{1}\right.$ 0years). Therefore large $\bar{\eta}_{F}$ would mean values greater than 25 (for $\nu=2$ ). For such values of the parameters the massive mode can be, more or less assumed to be stable.

One can also evaluate the lifetime for the $\Phi$ Yukawa coupling though qualitatively the results will not differ except for factors here and there.

\section{CONCLUSIONS}

Let us now summarise pointwise the results obtained in this article and briefly discuss some possible avenues of future research.

(i) In the presence of a sine-Gordon potential we have first discussed an exact solution for the warp factor and the scalar kink by solving the full set of Einstein-scalar equations in the presence of a negative cosmological constant. The background spacetime however, in this case, has a non-constant Ricci scalar which is asymptotically negative. The matter stress energy which generates this warped line element satisfies the null energy condition with isotropic negative pressure in the vicinity of the brane. The pressure along the extra dimension is, however, zero at the brane location and positive away from the brane.

(ii) Fermion fields have been studied in the background geometry of the exact solution mentioned in (i) above. We couple the scalar field to the spinor field through a Yukawa coupling and write down, following standard methods, the effective Schrodinger like equation for massive fermions. Two different types of Yukawa interactions are considered here, for 
each of which we analyse the issue of localisation of massive fermions. The difference between the effective potentials of the two Yukawa interactions lie in the shape and depth of the well at the brane location - for the same value of the Yukawa coupling parameter $\gamma_{F}$ the one which is more steep is prone to have more bound states. Using approximate methods we are able to obtain the maximum mass, the low-lying mass spectrum and the maximum number of trapped states for each case, for large values of the Yukawa coupling parameter. We also calculate the lifetime of these quasilocalised states and show that it can indeed be exponentially large for large $\gamma_{F}$ and moderate values of $\nu$. In addition, we also show that for zero or small values of $\gamma_{F}$ (fixed $\nu$ ) very few bound states may exist and the spectrum becomes almost continuous beyond these discrete states - a fact demonstrated through the vanishing of the level spacing for large values of the quantum number $n$.

For massless fermions, we verify previously known results. Only the left or the right chiral fermions can be localised under special restrictions on the value of the Yukawa coupling constant and other parameters that appear in the warp factor/kink solution.

(iii) An obvious question related to the background geometry arrived at in this paper is : is gravity localised on the brane in this model? The answer to this question, as far as we have been able to analyse, is in the affirmative. The potential felt by the graviton fluctuations is similar to the one in the RSII model with a well in the neighborhood of the brane followed by a barrier and a decay to zero as we go deep into the bulk. We shall elaborate on the localisation of gravity in our model in a future publication.

\section{Acknowledgments}

SK thanks S. Randjbar Daemi for discussions and the ASICTP, Trieste, Italy for support during part of the period when this work was done. RK thanks IIT Kharagpur for support.

[1] T. Kaluza, Sitzungsber. Preuss. Akad. Wiss., Phys-Math.Kl., Berlin Math. Phys., Bd. K1 966 (1921) ; O. Klein, Z. Phys. 37, 895 (1926)

[2] A. Salam and J. Strathdee, Ann. Phys. 141, 316 (1982); M. J. Duff, B.E.W. Nilsson and C. N. Pope, Phys. Rep. 130 C, 1 (1986); T. Appelquist, A. Chodos and P. G. O. Freund, Modern 
Kaluza-Klein Theories, Reading, MA, Addison-Wesley (1987)

[3] M. S. Green, J. H. Schwarz and E. Witten, Superstring theory, Cambridge University Press (1987); J. Polchinsky, String theory, Cambridge University Press (1997);

[4] L. Randall and R. Sundrum, Phys. Rev. Lett. 83, 3370 (1999); ibid Phys. Rev. Lett. 83, 4690 (1999)

[5] Y. Kim, C. O. Lee, I. Lee, J. J. Lee, hep-th/0307023 A. Padilla, hep-th/0210217 R. Dick, Class. Quant. Grav. 18, R1 (2001); C. Csaki, hep-ph/0404096

[6] B. Bajc and G. Gabadadze, Phys. Lett. B 474, 282 (2000); S. L. Dubovsky, V. A. Rubakov and P. G. Tinyakov, Phys. Rev. D 62, 105011 (2000)

[7] M. Besancon, Experimental introduction to extra dimensions, hep-th/0106165 ; Y. A. Kubyshin, Models with extra dimensions and their phenomenology hep-ph/0111027 and references therein.

[8] D. Langlois, gr-qc/0102007 (Proceedings of the 9th Marcel Grossmann meeting, July 2000 (Rome); gr-qc/0205004 (Proceedings of Journees Relativistes, Dublin 2001 and references therein ; E. Flannagan, S.-H. Henry Tye and I. Wasserman, Phys. Rev. D 62, 024011 (2000); H. Stoica, S.-H. Henry Tye and I. Wasserman, Phys. Letts. B 482, 205 (2000); J. S. Alcaniz , astro-ph/0202492

[9] W. D. Goldberger and M. B. Wise, Phys. Rev. D 60, 107505 (1999); H. Davoudiasl, J.L. Hewett and T.G. Rizzo, Phys.Lett. B473 43 (2000); S. C. Davis, JHEP 0203, 054 (2002); E. E. Flanagan, S.-H. Henry Tye and I. Wasserman, Phys.Lett. B522 155 (2001); B. Mukhopadhyaya, S. Sen, S. SenGupta, Phys.Rev. D65 124021 (2002); M. Gogberashvili, Phys.Lett. B553, 284 (2003); Rui Neves, Cenalo Vaz, hep-th/0302030

[10] C. Ringeval, P. Peter, J. P. Uzan, Phys. Rev. D 65, 044416 (2002); S. Ichinose, Phys.Rev. D66, 104015 (2002)

[11] For a useful summary on the details of the energy conditions see Lorentzian Wormholes : from Einstein to Hawking by Matt Visser (AIP, 1995)

[12] M. Gremm, Phys. Lett. B478, 434 (2000); Phys. Rev. D62,044017 (2000); K. Ghoroku and M. Yahiro, hep-th/0305150, A. Kehagias and K. Tamvakis, Mod. Phys. Lett. A 17, 1737 (2002),ibid. Phys, Letts. B504, 38(2001); M. Giovannini, Phys. Rev. D 64, 0106041 (2001), Phys. Rev. D 65, 064008 (2002); S. Kobayashi, K. Koyama and J. Soda, Phys. Rev. D65, $064014(2002)$ 
[13] O. DeWolfe, D. Z. Friedman. S. S. Gubser and A. Karch, Phys. Rev. D62 046008 (2000); C. Csaki, J. Ehrlich, T.J. Hollowood and Y. Shirman, Nucl. Phys. B581, 309 (2000) ; S. Ichinose, Phys. Rev. D 65,084038 (2002) ibid. Class.Quant.Grav. 18, 5239 (2001); A. Campos, Phys.Rev.Lett. 88141 (2002)

[14] M. Cvetic and H. Soleng, Phys. Rept. 282, 159 (1997); P.M. Cowdall, H. Lu, C.N. Pope, K.S. Stelle, P.K. Townsend, Nucl.Phys. B486 49 (1997); K. Skenderis and P. Townsend, Phys. Letts. B468, 46 (1999); K. Behrndt, Nucl.Phys. B573 127 (2000); i K. Behrndt, M. Cvetic, Phys.Lett. B475 253 (2000); M. Cvetic, H. Lu, C.N. Pope, Class.Quant.Grav. 174867 (2000); C. Csaki, J. Erlich, C. Grojean and T. Hollowood, Nucl. Phys. B584, 359 (2000); K. Behrndt, C. Herrmann, J. Louis, S. Thomas, JHEP 0101011 (2001); G. L. Cardoso, G. Dall'Agata, D. Lust, JHEP 0107026 (2001); A. H. Chamseddine, W. A. Sabra Nucl.Phys. 630326 (2002); C.M. Hull, JHEP 0111061 (2001); M. Bianchi, D. Z. Freedman, K. Skenderis , Nucl.Phys. B631, 159 (2002); K. Behrndt, M. Cvetic, Phys.Rev. D65 126007 (2002); L. Anguelova, C.I. Lazaroiu, JHEP 0209053 (2002); S. L. Cacciatori, D. Klemm, W. A. Sabra, JHEP 0303023 (2003)

[15] E Caliceti, V. Grecchi and M. Maioli, Commun. Math. Phys. 176,1 (1996), J. Zamastil, J. Cizek and L. Scala, Phys. Rev. Letts. 84, 5683 (2000); F. J. Gomez and L. Sesma, Phys. Letts. A 301, 184 (2002);; M. M. Nieto, Phys. Letts. B486,414(2000)

[16] C. M. Bender and T. S. Wu, Phys. Rev. 184, 5 (1969); C. M. Bender and T. S. Wu, Phys. Rev. D 7, 6 (1973); J. Zamastil, V. Spirko, J. Cizek, L. Skala and O. Bludsky, Phys. Rev. A 64, $042101(2001)$

[17] S. Randjbar-Daemi and M. Shaposhnikov, Phys. Letts. B492, 361-364 (2000)

[18] R. Jackiw and C. Rebbi, Phys. Rev. D13,3398 (1976); Y. Grossman, N. Neubert, Phys. Lett. B474 361 (2000).

[19] L.D. Landau and E.M. Lifshitz, Quantum Mechanics, Course of Theoretical Physics, Vol. 3, Third Edition (Butterworth bitemwb

[20] S. Weinberg, Gravitation and Cosmology (John Wiley and Sons, 1971) 